\title{
Management of Dysfunctional Catheters and Tubes Inserted by Interventional Radiology
}

\author{
Steven Y. Huang, MD ${ }^{1} \quad$ Bjorn I. Engstrom, $\mathrm{MD}^{2}$ \\ ${ }^{1}$ Department of Interventional Radiology, The University of Texas, MD \\ Anderson Cancer Center, Houston, Texas \\ 2 Division of Interventional Radiology, Consulting Radiologists LTD, \\ Minneapolis, Minnesota \\ ${ }^{3}$ Department of Radiology, Stanford University Medical Center, Palo \\ Alto, California \\ ${ }^{4}$ Division of Vascular and Interventional Radiology, Duke University \\ Medical Center, Durham, North Carolina \\ Semin Intervent Radiol 2015;32:67-77
}

\begin{abstract}
Address for correspondence Charles Y. Kim, MD, FSIR, Division of Vascular and Interventional Radiology, Department of Radiology, Duke University Medical Center, Box 3808, 2301 Erwin Road, Durham, NC 27710 (e-mail: charles.kim@duke.edu).
\end{abstract}

\author{
Abstract \\ Keywords \\ - catheter dysfunction \\ - tube obstruction \\ - interventional \\ radiology \\ - complications
}

Minimally invasive percutaneous interventions are often used for enteral nutrition, biliary and urinary diversion, intra-abdominal fluid collection drainage, and central venous access. In most cases, radiologic and endoscopic placement of catheters and tubes has replaced the comparable surgical alternative. As experience with catheters and tubes grows, it becomes increasingly evident that the interventional radiologist needs to be an expert not only on device placement but also on device management. Tube dysfunction represents the most common complication requiring repeat intervention, which can be distressing for patients and other health care professionals. This manuscript addresses the etiologies and solutions to leaking and obstructed feeding tubes, percutaneous biliary drains, percutaneous catheter nephrostomies, and drainage catheters, including abscess drains. In addition, we will address the obstructed central venous catheter.
Objectives: Upon completion of this article, the reader will be able to discuss how to diagnose and address common issues encountered with catheters and tubes placed by interventional radiologists.

Accreditation: This activity has been planned and implemented in accordance with the Essential Areas and Policies of the Accreditation Council for Continuing Medical Education (ACCME) through the joint providership of Tufts University School of Medicine (TUSM) and Thieme Medical Publishers, New York. TUSM is accredited by the ACCME to provide continuing medical education for physicians.

Credit: Tufts University School of Medicine designates this journal-based CME activity for a maximum of 1 AMA PRA Category 1 Credit $^{\mathrm{TM}}$. Physicians should claim only the credit commensurate with the extent of their participation in the activity.

Dysfunctional catheters and tubes, which often present with occlusion or leaking, are commonplace following minimally invasive percutaneous procedures. Without an organized approach, these complications can lead to increased patient morbidity and escalation of medical care with associated cost. Therefore, the interventional radiologist should be familiar with methods to establish the cause and potential solution(s) to dysfunctional catheters and tubes. In general, common principles hold true across a variety of catheters and tubes, and often similar solutions (such as catheter exchange) are frequently applied. However, there are important nuances that are specific to particular catheter types that can avoid additional procedures or outright catheter failure. The purpose of this review article is to describe systematic approaches to common dysfunctional catheters and tubes, including percutaneous feeding tubes, percutaneous biliary drainage (PBD) catheters, percutaneous catheter nephrostomies (PCNs), other drainage catheters including abscess drains and chest tubes, and central venous catheters (CVCs).
Issue Theme latrogenic Injury; Guest Editor, Baljendra Kapoor, MD, FSIR
Copyright $\odot 2015$ by Thieme Medical Publishers, Inc., 333 Seventh Avenue, New York, NY 10001, USA. Tel: +1(212) 584-4662.
DOI http://dx.doi.org/ 10.1055/s-0035-1549371. ISSN 0739-9529. 


\section{Percutaneous Enteral Feeding Catheters}

Percutaneous enteric catheters can be placed by open or laparoscopic surgical, endoscopic, or radiologic techniques. $^{1-3}$ Percutaneous radiologic placement of gastrostomy tubes (G-tubes), gastrojejunostomy tubes (GJ-tubes), and jejunostomy tubes (J-tubes) is safe and well tolerated. ${ }^{4,5}$ However, catheter revision for occlusion or tip malposition is not an uncommon event, occurring in up to $13 \%$ of G-tubes. ${ }^{5}$ This section discusses common problems of enteric catheters, specifically pericatheter leakage, catheter obstruction, dislodgement, malposition, and catheter-related pain, along with potential management options.

\section{Pericatheter Leakage}

Pericatheter leakage is a relatively common issue encountered with percutaneous G-tubes, GJ-tubes, and J-tubes. ${ }^{6,7}$ A small amount of leakage around the tube is common and not unexpected and may require up to two to three dressing changes per day. A greater degree of leakage is often problematic due to skin breakdown, as well as other considerations such as the demand of frequent bandage changes and soiled clothing/bedding. Feeding tube-related leakage can be difficult to control and results in chronic management issues for the patient.

There are several potential reasons for pericatheter leakage. Probably the most common reason is a tract that is not well sealed around the tube. Initially after percutaneous feeding tube insertion, the degree of sealing of the gastric wall or bowel around the tube is variable, usually with little to no associated leakage. Over the subsequent days and weeks after insertion, a foreign-body reaction to the tube occurs, consisting of acute and chronic inflammatory processes. ${ }^{8}$ After an initial inflammatory response, granulation tissue, consisting of fibroblasts and angioblasts in a matrix of collagen, begins to form around the foreign body (i.e., the catheter or tube), resulting in physiologic exclusion of the foreign body by means of fibrous capsule formation at the interface. This tract formation results in a sleeve of fibrous tissue encapsulating the tube circumferentially along its length. As the tract continues to mature over time, tract contraction results in sealing around the tube with cessation of leakage under normal circumstance. However, as this process requires normal healing mechanisms, patients with malnutrition, malignancy, or systemic inflammatory states may not have normal healing, and thus expected tract maturation and sealing may not occur-and in some cases, the tract may enlarge. Unfortunately, patients with malnutrition, malignancy, and other systemic pathologies are those who often need percutaneous enteral feeding access.

Another cause of pericatheter leakage is excessive gastric pressurization related to gastroparesis, ileus, or obstruction, which can force gastric or enteric fluid through the tract around the tube. Another potential etiology for leakage around the tube is a fistula from the gastric lumen to the tract. In the case of GJ-tubes, leakage of tube feeds around the catheter may be a sign that the jejunal limb has retracted into the stomach as cause for pressurization. An abdominal radiograph can be useful for ascertaining whether this has occurred (-Fig. 1). Since GJ-tubes are typically inserted in lieu of G-tubes due to a contraindication to gastric feeding, this catheter malposition should be promptly addressed. J-tubes have been shown to have a higher rate of pericatheter leakage. ${ }^{4}$ This may be related to the thinner jejunal wall when compared with stomach wall or lesser luminal capacity that may be more prone to the effects of ileus or dysmotility.

\section{Buried Bumper Syndrome}

Buried bumper syndrome is characterized by erosion of the internal retention bumper or balloon into the stomach or bowel wall. Essentially all percutaneous enteral feeding tubes can manifest with buried bumper syndrome, although this entity is by far most often described with endoscopically inserted G-tubes. Excessive or chronic pressure exerted upon the gastric wall and abdominal wall in between the internal bumper or balloon and the external retention disc can induce ischemia of the intervening structures with breakdown of tissues. The reported incidence is as high as $21.8 \%$ of cases, ${ }^{9,10}$
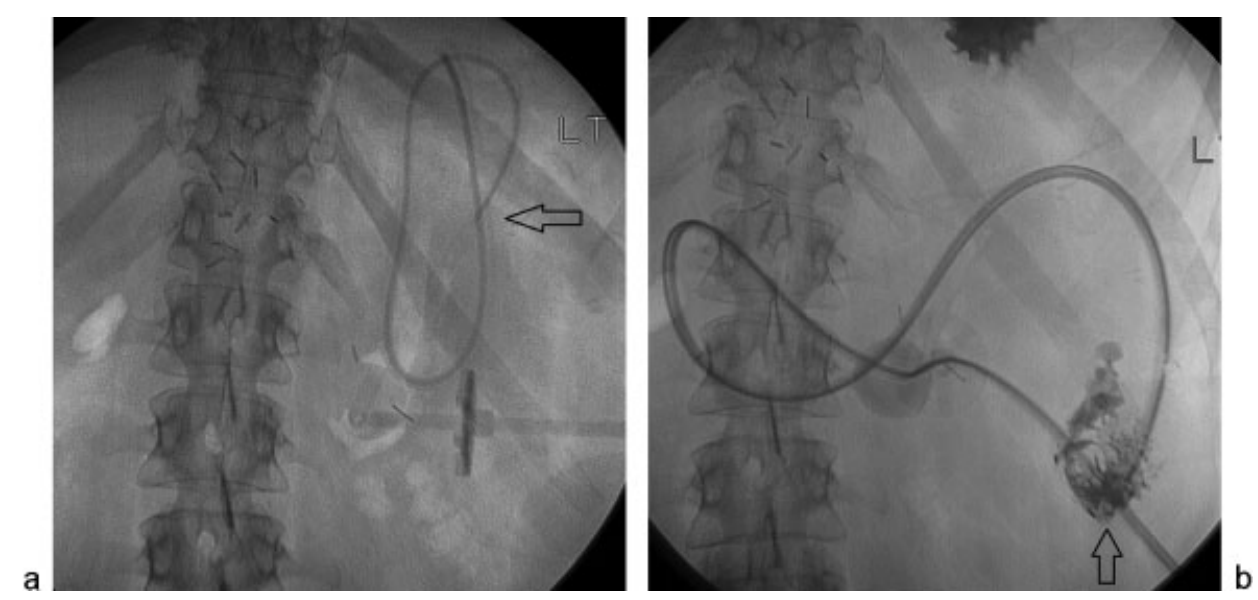

Fig. 1 G-tube malposition. A 68-year-old man with gastroparesis presenting with bloating during tube feeds into the jejunal port with pericatheter leakage of tube feeds. (a) Initial scout radiograph demonstrates the tube to be coiled over the left upper quadrant in an abnormal configuration (arrow). (b) The GJ-tube was exchanged for a new one, in appropriate position as confirmed by contrast injection into the jejunal port (arrow). 
and typically first presents between 3 and 6 months postinsertion. ${ }^{11}$ This clinical finding ranges from simple ulceration underneath the internal bolster, to an enlarging hole around the tube, to complete erosion of the retention bumper or balloon through the anterior abdominal wall with a resulting large defect. ${ }^{12}$ Severe cases of buried bumper syndrome can result in abscess formation, necrotizing cellulitis, or, rarely, tube failure as the tract becomes sealed off and reepithelialized on the gastric mucosal side. ${ }^{10}$

\section{Management of Skin Breakdown Associated with Pericatheter Leakage}

Since gastric and intestinal contents are caustic to the skin due to the low $\mathrm{pH}$ and enzymes, skin breakdown often occurs in the setting of chronic leakage, which can eventually result in large skin defects. Thus, cessation of leakage is important to allow healing. Furthermore, the low pH and enzymatic activity may impair tract maturation and cause tract enlargement, resulting in worsening of leakage and continued tract enlargement. Thus, attention to wound care is an important aspect in the management of pericatheter leakage. Ostomy and wound care nurses typically have the ideal expertise to manage wounds related to enteric contents. Local measures such as powdered absorbing agents or a skin protectant such as a paste of zinc oxide may be used. A pectin powder can be sprinkled onto the irritated surface, followed by a saturating layer of liquid skin barrier, to protect the skin from further breakdown related to enteric fluids and facilitate healing. In difficult cases, an ostomy pouch may be ideal for protecting the skin around the defect and allowing convenient collection of the leaking fluid. In these cases, a customized pouch should be used, allowing the tube to exit the pouch with sealing around the tube. Proton-pump inhibitors may also be useful for decreasing the caustic effect of leaking contents on skin.

\section{Management of Pericatheter Leakage}

When pericatheter leakage is due to excessive gastric pressurization, gastric distention can be usually visualized on abdominal radiography or computed tomography (CT). In these cases, venting the G-tube to gravity or suction on a constant or intermittent basis may resolve the leakage, but will need to be continued until the underlying etiology has resolved. $^{13,14}$

In patients with a balloon-retained or bumper-retained feeding tubes, very mild traction on the tube allowing the balloon or bumper to abut the intragastric portion of the tract can help to decrease leakage. However, cinching the external retention with excessive tension can induce ischemia of the intervening structures and impair tract healing (buried bumper syndrome). ${ }^{12}$ New pericatheter leakage with balloon retention feeding tubes presenting in the setting of new tube instability may also indicate that the balloon has ruptured. In this scenario, the patient should secure the tube to the skin with tape and subsequently undergo tube exchange. Similarly, if the tube is a multi-side-hole catheter, a side hole may have retracted within the tract. In this case, catheter advancement or exchange in the setting of a broken retention suture is indicated.
To solve the problem of pericatheter leakage, some interventionalists may attempt to place a larger diameter gastrostomy tube with the thought of minimizing space around the tube for leakage to occur. Exchanging the tube for a larger diameter can help seal the gastrostomy tract temporarily, but is not recommended as it often results in eventual tract enlargement with worsening leakage. ${ }^{12}$ If the tube functioned well for a time after placement without pericatheter leakage, it may be reasonable to remove the catheter altogether to allow for the tract to partially heal and contract. With daily monitoring of the old tract diameter, once the tract has sufficiently contracted, the patient can undergo catheter replacement via the same access site.

Fistulas between the gastric or bowel lumen and the fibrous tract are extremely difficult to identify and may be difficult to resolve. In cases of persistent or severe pericatheter leakage of any etiology that is refractory to all attempts, tube removal may be the only viable option. Tube insertion at a different site could be performed, but the underlying issues causing persistent leakage may be encountered.

For GJ-tubes where the jejunal limb has migrated retrograde into the stomach, suboptimal tract angulation may be responsible for retrograde tip malposition, particularly when a G-tube was originally inserted endoscopically with subsequent conversion to a GJ-tube. ${ }^{15}$ The tube could be addressed by reinserting a new GJ-tube appropriately into a postpyloric position-however, if suboptimal tract angulation is present, the patient may be at risk for recurrent malposition. Alternative options include removing the GJ-tube and inserting a new one with a more optimal tract angulation or abandoning gastrojejunal feeding access for J-tube access.

\section{Feeding Catheter Obstruction}

Catheter obstruction is another common problem that is typically related to inadequately crushed medications or inadequate flushing, which allows feeds or medications to accumulate and become impacted within the tubing. ${ }^{16}$ of paramount importance in preventing this phenomenon are explicit patient and provider instructions. Pills that must be administered through the tube must be finely crushed and diluted into a large volume of fluid. The tube should be flushed with 30 to $60 \mathrm{~mL}$ of fluid before and after tube feeds. In the setting of continuous tube feeds, intermittent flushing every 3 to 4 hours is also recommended for patients with issues with tube obstruction. Excessive intraluminal yeast colonization has also been associated with recurrent episodes of tube obstruction. ${ }^{17}$

\section{Management of Feeding Tube Obstruction}

Irrigation of the obstructed tube with saline or water is an appropriate initial step for obstructing feeding tubes. ${ }^{16}$ If gentle flushing of the tube with fluid does not work, a more forceful injection may be effective to eject the obstructing material. Care must be taken, however, since excessive force could easily result in tube rupture at any point along the tube. A carbonated beverage can be infused and allowed to dwell in the tube for some time before attempting to flush again. A solution containing a pancreatic enzyme mixture (Viokase, 
Mont Saint-Hilaire, Quebec, Canada) has been reported to have a better effect than other solutions. ${ }^{16}$ An alkalinized pancreatic enzyme (Creon, AbbVie Inc, Chicago, IL) was effective in clearing approximately half of the occluded enteral feeding tubes in a recent retrospective study, which is an efficacy rate much lower than that previously reported in the literature with a Viokase-based protocol. ${ }^{18}$

When catheter obstruction is refractory to conservative methods, catheter exchange is often needed. For G-tubes with a well-matured tract (more than 3 months), simply removing the tube and reinserting can be a quick and effective solution. If there is any uncertainty as to the tract maturity, or for all GJ-tubes, tube exchange over a guidewire should be performed. Inserting guidewires through obstructed catheters can be highly challenging. A stiff hydrophilic guidewire tends to be more effective, although gastroenteric contents tend to make hydrophilic wires very sticky; frequent rehydration of the hydrophilic coating is often necessary. In cases when it cannot be advanced through the lumen of the catheter, it may be possible to advance the guidewire along the outside of the catheter. For pigtail loop retention mechanisms, a peel-away sheath, sized 1 French larger than the catheter, can be advanced over the catheter, allowing maintenance of luminal access after removal of the catheter. In cases of repeated episodes of obstruction, catheter upsizing should be considered. In cases when all measures have failed, a decision must be made as to whether the tube can be safely removed with tract renegotiation using a guidewire and catheter - if not, then waiting an appropriate time interval until tract maturation is reasonable.

\section{Catheter Migration/Malposition}

Gastric peristalsis can result in forward migration of gastrostomy tubes beyond the pylorus when they are not well retained to the skin by the retention mechanism or suture. Patients will most often present with feeding intolerance with bolus feeds infused into the duodenum and/or excessive gastric distention that is unresponsive to gastric suctioning. The catheter can usually be retracted and repositioned without much difficulty (-Fig. 2). When balloon-retention catheters migrate to a peripyloric position, patients may present with gastric outlet obstruction. ${ }^{19}$

Management of gastrostomy tubes found to be completely misplaced (e.g., intraperitoneal or transcolonic) depends on patient presentation. If tube feeds have been infused intraperitoneally, patients should be started on broad-spectrum antibiotics and evaluated by the surgical team for potential peritoneal washout. Catheters placed into the peritoneum can often be removed without any clinical sequelae. If there are any defined fluid collections, image-guided aspiration and drain placement is recommended. In the event of inadvertent transcolonic access, the gastrostomy catheter should not be removed until tract maturation has occurred to decrease the risk of peritoneal spillage. Both surgical and conservative management strategies have been successful in this setting. ${ }^{20,21}$

\section{Catheter Dislodgement}

Accidental dislodgement or removal of enteric catheters is a common complication, with a reported estimated frequency
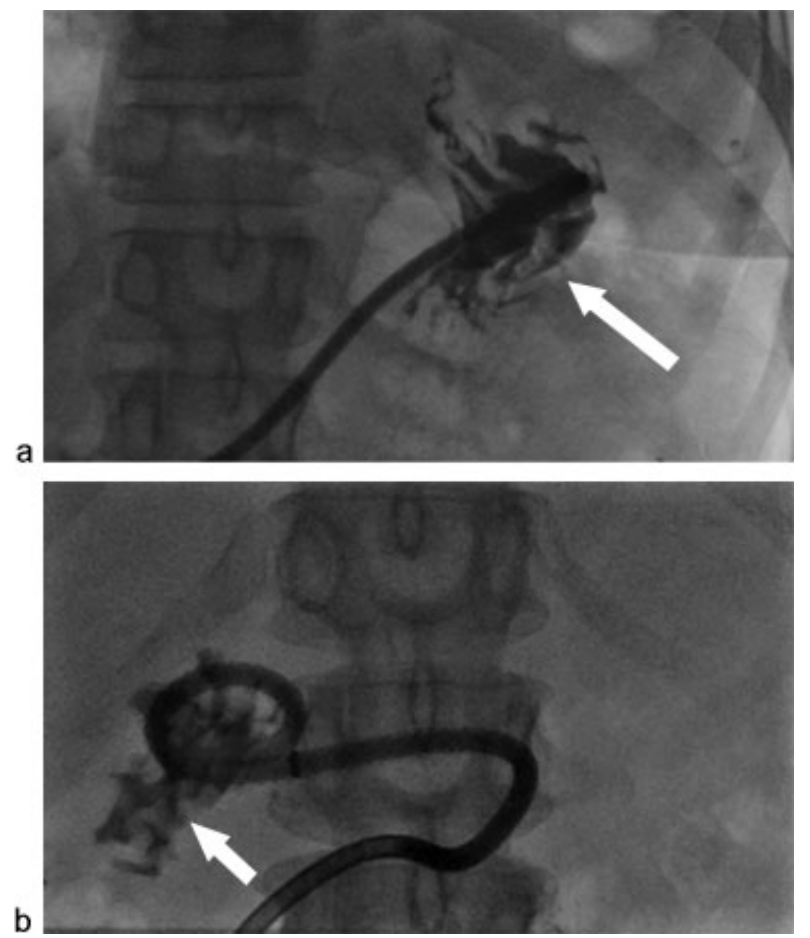

Fig. 2 Migration of Cope-loop locking pigtail-type feeding gastrostomy catheter. (a) Fluoroscopic image immediately following gastrostomy tube placement. Contrast injection confirms intragastric positioning (arrow). (b) The patient complained of new-onset intolerance to bolus tube feeds 3 months after gastrostomy placement. Fluoroscopic image demonstrates gastrostomy catheter migration; contrast injection confirms postpyloric positioning with opacification of the duodenum (arrow).

of $11.9 \%{ }^{22,23}$ To prevent catheter dislodgement, enteric feeding tubes often have retention mechanisms such as a rubber bumper, balloon, or pigtail loops inside the gastric lumen. Catheters may become dislodged during sleep, normal daily activities, or self-removal in cases of disoriented patients.

While tube dislodgement is usually a relatively benign complication, the consequences can be more severe in the setting of recently inserted tubes without concomitant gastropexy or jejunopexy (typically endoscopically inserted tubes). In the setting of tube dislodgement before tract formation and without gastropexy for G- or GJ-tubes or jejunopexy for J-tubes (i.e., $<4$ weeks after insertion), the stomach or jejunum can pull away from the anterior abdominal wall, with resulting spillage of enteric contents into the peritoneal cavity. Physical examination for an acute abdomen and/or CT can help determine whether surgical management is needed (-Fig. 3). While the primary purpose of T-fastener gastropexy is to facilitate tube insertion, the presence of T-fasteners may also facilitate regaining gastric access in the setting of early inadvertent tube dislodgement., 24,25 However, a study of T-fastener location after gastropexy revealed that $41 \%$ of $\mathrm{T}$-fasteners had detached intraluminally or had migrated within the abdominal wall based on CT scans performed within 1 month of gastropexy. ${ }^{26}$

For enteral feeding tubes with mature tracts, reinsertion of enteral feeding tubes can usually be easily performed if the patient presents soon after dislodgement. For well-developed 


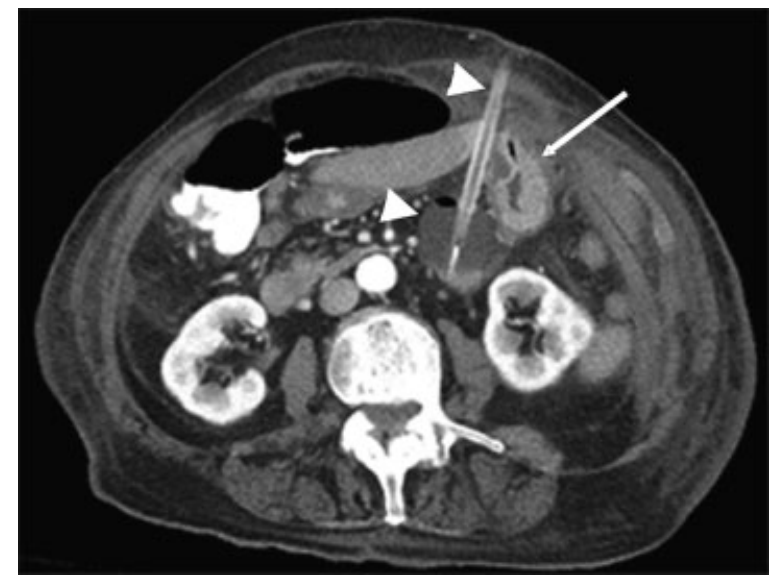

Fig. 3 Gastrostomy tube malposition. A 92-year-old disoriented woman removed a gastrostomy tube 2 days after placement. A physician in the emergency room attempted replacement with a Foley catheter. Given severe abdominal pain and immature tract, a CT scan was performed. Axial CT image demonstrates a misplaced Foley catheter (arrowheads) in the peritoneum adjacent to the stomach (arrow). The catheter was subsequently removed, and the patient was placed on broad-spectrum antibiotics and successfully managed conservatively.

tracts, G-tubes can often be safely directly reinserted. However, if there is any concern for catheter position, a tube injection under fluoroscopy or with subsequent X-ray should be performed prior to enteral feeding. For more difficult G-tube cases and for all GJ- and J-tube cases, insertion of a guidewire through the tract should be performed, with advancement of the tube over the guidewire. The success rate for reinsertion of G- and GJ-tubes through an established tract is reported to be $91 \%$ ( 156 or 170 ), with decreasing rates of success with longer durations after dislodgement. ${ }^{22}$ The success rate is high if reinserted within 3 days after dislodgement; reinsertion attempts performed 4 or more days after dislodgement had a lower (71\%) success rate. ${ }^{22}$ Feeding tubes that had been indwelling for longer periods of time were also associated with improved success rates.

When G-tube reinsertion is needed for patients with a healed tract, needle access at the prior site is often easily performed without need for repeat gastropexy. For re-access of J-tubes at a healed site, ultrasound can be used to visualize the tract to allow repuncture of the jejunum through the tract, with a reported $92 \%$ success rate. ${ }^{27}$

\section{Percutaneous Biliary Drainage Catheters}

Biliary drainage procedures are associated with significant catheter-related complications, occurring in up to $22 \%$ of procedures. $^{28}$ Complications affecting catheter function include pericatheter leakage, catheter obstruction, postclamping cholangitis, catheter obstruction, and catheter dislodgement/malposition. In this section, etiology, prevention, and management of these complications are discussed.

\section{Percutaneous Biliary Drainage Obstruction}

PBD catheter obstruction is usually the result of bile stones, biliary sludge, blood clots, or intestinal debris. Daily flushing of the catheter with saline can help prevent obstructive episodes. Signs of PBD obstruction include rising serum bilirubin levels, right upper quadrant pain, fever, leukocytosis, jaundice, pruritus, nausea, abrupt decrease in catheter output (for those on external drainage), resistance with flushing, and/or pericatheter leakage. If any of these findings are encountered, catheter obstruction must be considered and promptly addressed. Since the presence of a PBD across the sphincter of Oddi results in biliary colonization with intestinal bacteria, obstruction of the catheter (and biliary tree) can rapidly result in cholangitis and progress to sepsis if not recognized and addressed in a timely fashion. Cholangiography via the PBD catheter can demonstrate patency of the catheter and side holes; however, partial obstruction can be difficult to ascertain. Therefore, empiric catheter exchange is commonly performed in the setting of signs or symptoms that suggest the possibility of PBD obstruction. If catheter exchange plus empiric antibiotics do not mitigate these signs or symptoms, a search for an alternative cause is warranted. In cases of recurrent obstruction, PBD upsizing to 12 or 14 French (or larger) may be helpful. In rare cases, catheter kinking or an excessively tight skin anchoring suture may be the cause of catheter obstruction.

\section{Pericatheter Leakage}

Pericatheter leakage encountered with PBDs is highly suggestive of catheter obstruction, and thus should be addressed as described above. When the catheter is obstructed and no longer decompressing the bile ducts, intrabiliary pressure increases and eventually forces bile to travel along the path of least resistance, which is usually along the tract on the outside of the catheter. Another cause for pericatheter leakage is catheter malposition. The multiside-hole biliary drainage catheter should be ideally positioned so that there are side holes above and below the level of obstruction. If the catheter has migrated toward the bowel, such that all side holes are downstream from the obstruction, the biliary tree remains obstructed and there is leakage of bile along the catheter tract. ${ }^{28}$ Conversely, if the catheter is retracted enough that side holes are outside of the biliary tree but within the tract, bile can easily leak externally around the catheter. In all of these cases, catheter exchange is warranted, with special attention to catheter positioning and anchoring at the skin. Only rarely will pericatheter leakage occur in the absence of obstruction or catheter malpositioning; in these cases, catheter upsizing can be considered. In patients with ascites, pericatheter leakage of ascitic fluid can be mistaken for bile. In these cases, drainage of peritoneal fluid on a transient basis (needle paracentesis) or a chronic basis (tunneled peritoneal drainage catheter) can be performed until tract maturation occurs.

\section{Percutaneous Biliary Drainage Dislodgement/ Migration/Retraction}

PBD catheter dislodgement is reported in up to $3.4 \%$ of cases. ${ }^{29}$ PBD catheters are typically held in place by a suture anchor at the skin site, the internal retention loop in the 
duodenum, and any additional external fixation devices or tape. External biliary drainage catheters, which are used when the biliary occlusion cannot be traversed, require particular attention to the securement, since these will very easily become displaced or retracted. When catheter retraction occurs, patients should be instructed to tape the catheter in place and should undergo a fluoroscopic catheter evaluation as soon as reasonably possible. In some cases, catheter retraction is suspected but uncertain because the retaining suture is intact but may have slipped. In this case, new pericatheter leakage, persistent pain, and alterations in biliary catheter output (for externally draining catheters) are supportive of the diagnosis of catheter malposition. Initial evaluation can include an abdominal radiograph, with comparison to the postprocedural image to ascertain whether significant catheter retraction has occurred. If uncertain, contrast injection under fluoroscopy is indicated.

While physical traction on the catheter is the most common cause for PBD retraction, spontaneous retraction or migration can also occur. Catheter migration can occur due to respiratory motion and routine movement, even when the catheter is securely anchored to the skin. Essentially, the catheter can loop into the perihepatic space, with retraction of the pigtail loop toward or into the common bile duct. This phenomenon is more prone to occur in newly inserted catheters prior to tract formation. Migrated catheters may present with pain due to irritation of the adjacent peritoneum and/or catheter obstruction if kinking occurs. PBD retraction can also occur in the setting of morbid obesity, where the mobility of the pannus and skin can retract the catheter due to the suture anchor to the skin. This may be more prone to occur for right-sided catheters placed at the lower aspect of the ribcage due to the relatively higher degree of subcutaneous thickness and pannus mobility. In these cases, a left-sided approach placed in the subxiphoid region may provide more catheter stability, or if a right-sided catheter is necessary, a higher and more anterior site may be optimal. Furthermore, avoidance of catheter anchoring at the exit site can be considered, with primary reliance on the internal pigtail loop for catheter retention.

If PBD catheter dislodgement occurs in the setting of a mature tract, catheter reinsertion can be attempted with high success rates if performed within 2 days of dislodgement. ${ }^{22}$ An initial tractogram performed by pressing a blunt syringe tip into the exit site can be helpful to delineate the presence and configuration of the tract. It is ideal to avoid or minimize injection of local anesthetic at the exit site, which could disrupt or narrow the tract. Using a catheter and guidewire combination, attempts can then be made to negotiate the tract into the biliary system. Hydrophilic guidewires tend to be better suited for this purpose. In certain cases, however, de novo access may be required. ${ }^{28}$

\section{Percutaneous Catheter Nephrostomy}

PCN has become an essential interventional procedure in the management of patients with various urinary tract diseases such as ureteral obstruction due to both benign and malignant causes; it is also used for patients with ureteral or bladder leaks due to various causes. ${ }^{30}$ At present, the most commonly used PCN catheter is a self-retaining pigtail catheter with a locking string, which contains either a single- or double-stranded cord inside the lumen of the catheter. ${ }^{31}$ After placement has been achieved, routine exchange of the nephrostomy catheter is required to prevent obstruction related to mineral deposition and encrustation with risk for sepsis. The procedure is frequently completed in a few minutes, often without sedation. Routine nephrostomy exchange is typically performed at 1 to 4 month intervals.

\section{Percutaneous Catheter Nephrostomy Obstruction}

PCN obstruction is usually secondary to mineral encrustation within the lumen of the catheter. In patients with hematuria, blood clots can also result in tube obstruction. Patients will present with decreased or cessation of urine output, pericatheter leakage of urine, flank pain, fever, and/or sepsis. Similar to PBD obstruction, PCN obstruction warrants that catheter exchange be performed as soon as reasonably possible due to the risk of sepsis, with consideration for upsizing. Advancement of a guidewire through the nephrostomy catheter can be quite challenging in the setting of catheter encrustation. In cases when conventional and hydrophilic guidewires are unsuccessful, several additional approaches can be taken:

- Peel-away sheath technique. The existing PCN is cut, while maximizing the length of the remaining catheter. A peelaway sheath that is the same size or $1 \mathrm{~F}$ larger is then advanced over the PCN into the collecting system. If there is inadequate catheter length to reach through the peelaway sheath, a suture is tied to the proximal cut end of the indwelling PCN and brought through a peel-away sheath. The PCN is then pulled out. A new tube can be inserted through the sheath; use of a guidewire may be helpful in forming the pigtail loop.

- "New side hole technique." A metal cannula with a blunt stylet (such as the Hawkins needle) is advanced into the nephrostomy tube to the point of obstruction. The blunt stylet is then replaced with a sharp stylet and advanced a short distance through the obstructed catheter and the stylet is then removed. Contrast is then injected to confirm positioning in the renal pelvis, followed by advancement of a 0.035 " wire for secure access. The obstructed pigtail can then be more forcefully removed through the sheath while maintaining wire access. ${ }^{32}$

- Pericatheter technique. Either a guidewire alone or a guidewire inserted through a small 5 French catheter is advanced into the tract alongside the occluded catheter. Success of this maneuver depends on maturation of the tract. A floppy-tip or J-tipped guidewire is preferred, to avoid damage to or perforation of the tract. Negotiation of the length of the tract may be difficult if areas of tortuosity or stricture are present. Injection of contrast material through the catheter will allow depiction of the route necessary for cannulation. ${ }^{33}$ However, renal parenchyma 
may be tightly sealed around the catheter leading to difficulty entering the pelvis or possibly the creation of false channels. ${ }^{34}$

\section{Pericatheter Leakage}

Similar to PBDs, a leaking PCN usually indicates the catheter is either obstructed or retracted partially or fully into the tract. Catheter exchange is indicated.

\section{Percutaneous Catheter Nephrostomy Dislodgement| Malposition}

PCN displacement is among the most common complications of PCNs, with most studies citing rates of around $15 \%{ }^{35}$ In cases where the PCN has been completely dislodged, there is often a limited window of time available to replace the catheter into the existing tract. Success rates for reinsertion vary, but one study of 25 PCN reinsertions reported an $88 \%$ reinsertion success rate when performed within the first 48 hours after dislodgement. ${ }^{22}$ In cases where there is continued leakage of urine, the tract is usually still intact and easily traversed with a catheter and hydrophilic guidewire. Similar to PBD reinsertions, a tractogram can be attempted, and local anesthesia should be used sparingly. Not infrequently, the exit site may appear to be healed without a visible orifice. However, if a scab is present, unroofing of the scab may allow visualization and access to the origin of the tract. Techniques to replace a catheter via an existing tract vary widely, but most commonly involve the use of a 4 to 5 French catheter with a hydrophilic guidewire. The guidewire is used to gently probe the tract with concomitant advancement of the catheter, taking particular care not to create a false passage, until the collecting system is reached.

Similar to PBD catheters, nephrostomy catheters may be inadvertently retracted. Symptoms of malposition include a sudden decrease in output, pain, bloody urine, pericatheter leakage, and fever (from obstruction). An abdominal radiograph can be performed to assess catheter configuration with comparison to prior images. An abnormally configured catheter loop, particularly one of diminished diameter, should raise suspicion that the catheter has been retracted into the tract ( - Fig. 4). Again, if there is any concern, a catheter check and exchange should be performed under fluoroscopy.

\section{Abscess Drainage Catheter Dysfunction}

As with the other percutaneous drainage catheters above, pericatheter leakage of abscess drains are usually due to catheter obstruction or malposition, thus requiring a drainage catheter injection and catheter exchange. In the case of viscous fluid, catheter upsizing should be considered. In the setting of intra-abdominal abscess drains, a bowel leak should be suspected if continued high output and pericatheter leakage is encountered. Similarly, persistently high output of pseudocyst drainage catheters may indicate communication with the pancreatic duct. ${ }^{36}$ Prolonged use of catheters in this scenario may allow diversion of enteric contents away from healing wounds with the aid of a vacuum-assisted wound closure device. When healing of the incision/wound has taken place, attempts can be made at gradually downsizing the tube to reduce the amount of secretions while allowing for tissues to scar down around the tube. Working closely with the surgeon is a must in this scenario, as this is a very difficult problem to resolve and may require a reoperation.

\section{Central Venous Catheter Dysfunction}

CVCs are commonly inserted and managed by interventional radiologists. While the procedural complexity is generally low, the subsequent management of catheter dysfunction is more complex.

\section{Central Venous Catheter Occlusion}

Occlusions are among the most common complications associated with CVC maintenance, characterized by the
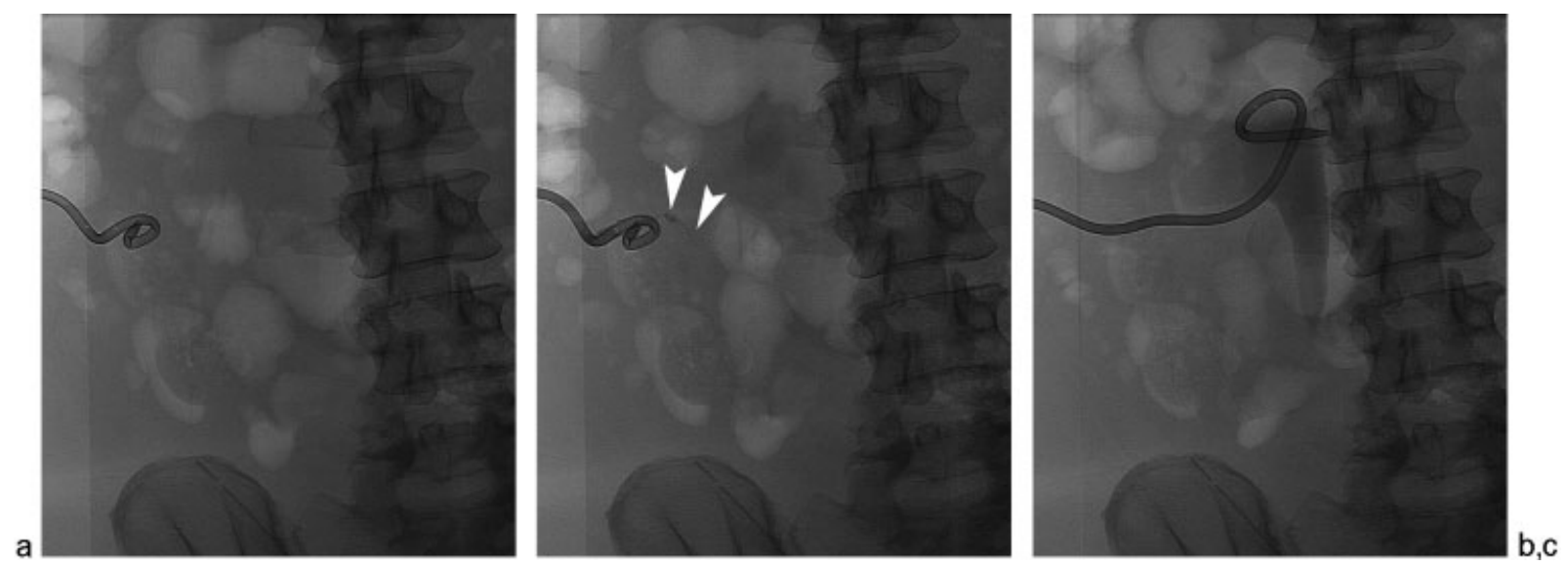

Fig. 4 PCN malposition. A patient with a chronically indwelling left PCN presented with new pericatheter leakage of urine. (a) Initial fluoroscopic image demonstrates an abnormally small-diameter PCN pigtail loop that is somewhat more lateral than is usual for the renal pelvis. (b) Injection of the catheter demonstrates subtle opacification of the tract (arrowheads) with flow of contrast back along the catheter tract with minimal collecting system opacification, consistent with the PCN having been retracted into the tract. (c) After negotiation of the tract with a catheter and guidewire, a new PCN was inserted with pigtail loop appropriately positioned in the renal pelvis. Notice the much larger (normal) diameter of the pigtail loop and more medial position. 
inability to aspirate blood from the lumen of a catheter. Studies in pediatric and adult patients suggest that occlusions occur in up to $36 \%$ of CVCs within 2 years of placement. ${ }^{37}$ The most common causes of CVC occlusion are intraluminal thrombus formation and/or fibrin sheath formation around the tip of the catheter. ${ }^{38}$ Pericatheter fibrin sheath formation has been demonstrated to occur as early as 24 hours after placement and is thought to occur in 80 to $100 \%$ of CVCs in the first week after insertion. ${ }^{39}$ Additional etiologies for occlusion include catheter kinking, an excessively tight anchor suture (-Fig. 5), and catheter tip malposition, such as retraction of the tip into the tract, retraction into an occluded or stenotic venous segment, or migration of the tip into a small branch vein.

The initial step in the evaluation of a dysfunctional CVC is a chest radiograph, to identify catheter kinking or catheter tip malposition (-Fig. 6) ${ }^{40}$ If either of these is present, catheter exchange or revision is indicated. If the radiograph is unremarkable, an attempt at dissolution of presumed thrombus within the catheter lumen or fibrin sheath at the catheter tip can be attempted. ${ }^{41,42}$ One suggested dose regimen is the injection of $1.0 \mathrm{mg}$ tissue plasminogen activator (tPA) in each lumen, which is then allowed to dwell over at least 1 hour. ${ }^{41}$ If tPA dwelling results in a restored ability to aspirate blood from the lumen, the catheter can continue to be used; if tPA is ineffective or deemed inappropriate, catheter evaluation under fluoroscopy is warranted. In this instance, injection of contrast into the catheter should be performed to identify the etiology of dysfunction, such as a fibrin sheath, venous occlusion around the catheter tip by stenosis or thrombosis, or catheter tip malpositioning against the vein wall or in a small branch vein. If a fibrin sheath is present, contrast will

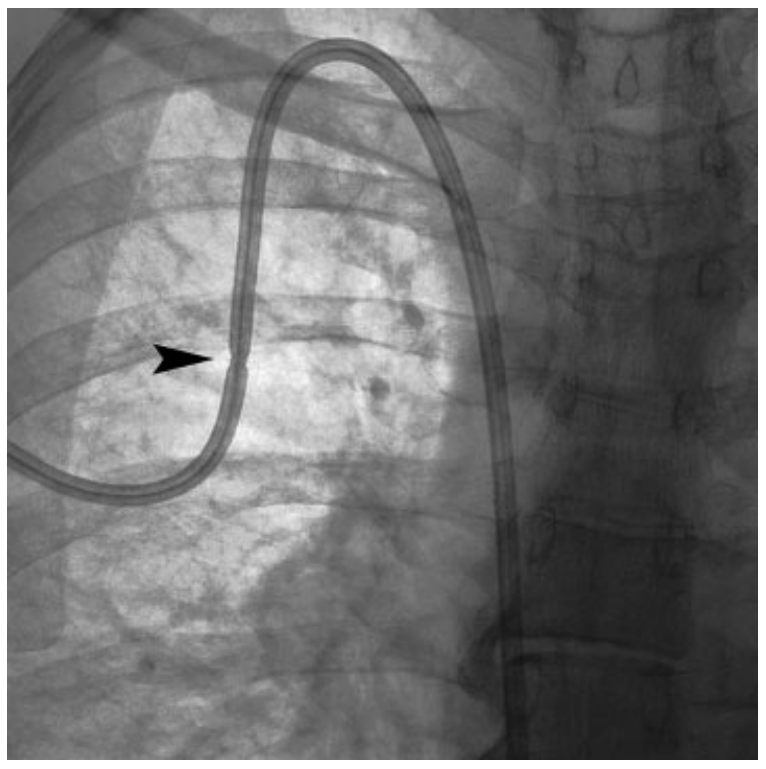

Fig. 5 A 59-year-old woman with multiple myeloma underwent triple lumen Hickman catheter for plasmapheresis, presenting the next day with a dysfunctional catheter. The initial chest radiography demonstrates an indentation at the catheter anchor suture (arrowhead). After this was released and resutured with less tension, the catheter functioned well.

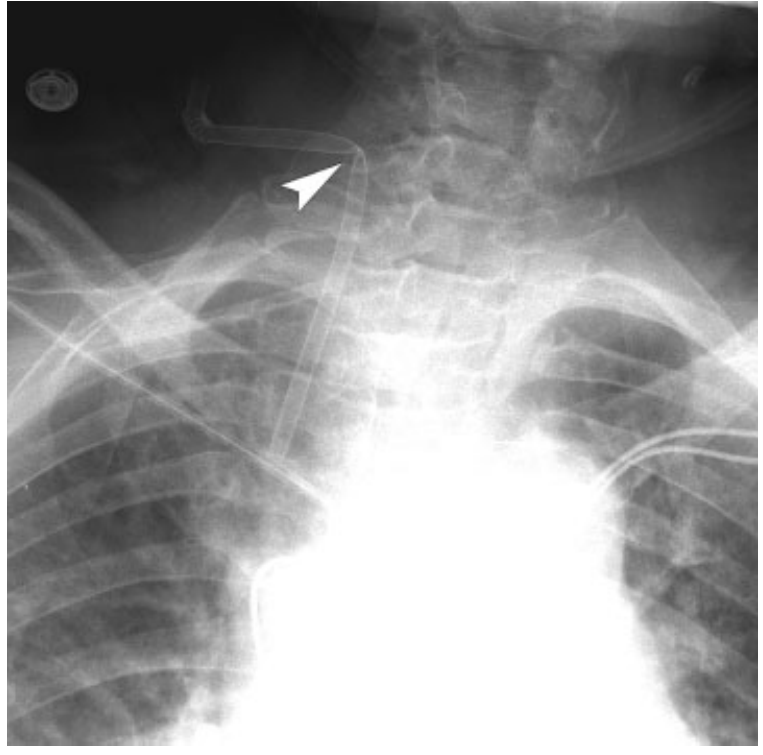

Fig. 6 Inability to aspirate from a right jugular catheter. The catheter is clearly kinked (arrowhead) as the likely cause for inability to aspirate blood.

accumulate around the catheter tip and travel retrograde along the outer surface of the catheter, exiting the fibrin sheath through one or more perforations (-Fig. 7). The usual dissemination of contrast out of the catheter tip will not be noted if a fibrin sheath is present.

If catheter injection demonstrates a fibrin sheath, fibrin sheath disruption can be considered. ${ }^{43,44}$ For tunneled catheters, this involves removal of the catheter over a guidewire, then advancing an angioplasty balloon (10-12 mm diameter) over the guidewire, stopping several centimeters short of the

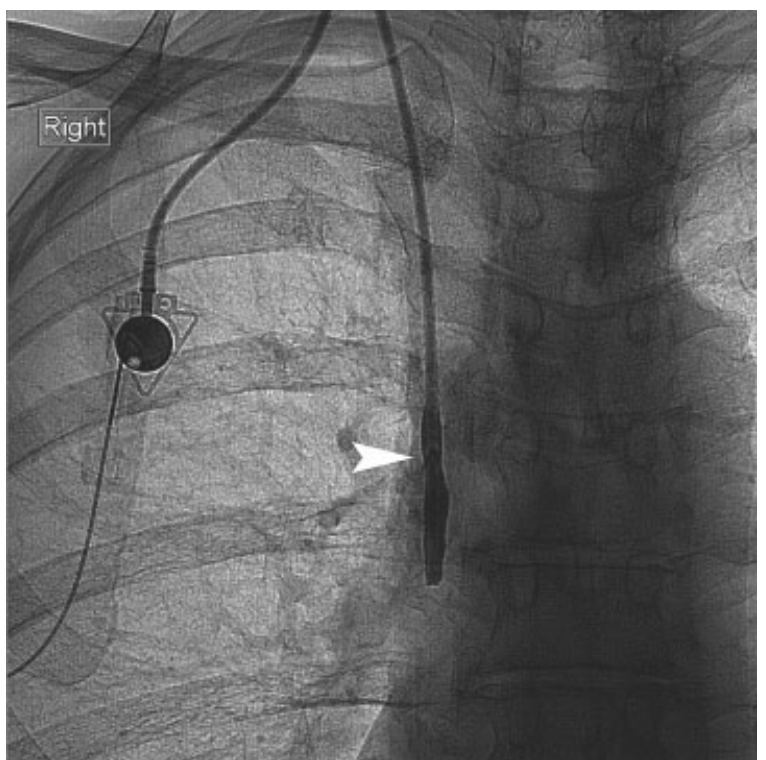

Fig. 7 Fibrin sheath formation around the port catheter as cause for dysfunction. During injection of contrast into the port reservoir, note the accumulation of contrast around the catheter with lack of a jet of contrast out the catheter tip. Also, note the focal jet of contrast through a perforation in the fibrin sheath (arrowhead). 
prior catheter tip position. The balloon is then inflated to its maximum diameter. This theoretically results in separation of the fibrin sheath at that site. The balloon is then advanced over the guidewire for several centimeters. This maneuver should theoretically avulse the distal fibrin sheath segment. When the new catheter is reinserted over the guidewire, the tip will be completely free of the residual fibrin sheath, although fibrin sheath will still be encapsulating the segment of catheter near the venotomy. Alternatively, advancing the catheter at least $1 \mathrm{~cm}$ deeper than its original position will also achieve this effect, if the catheter tip is not already at its maximum depth in the right atrium. For left-sided catheters, advancement of the catheter into the mid-to-deep right atrium will provide optimal functionality. ${ }^{45}$ For tunneled hemodialysis catheters, fibrin sheath stripping was shown in a randomized clinical trial to be less effective than overthe-wire catheter exchange for long-term patency and is thus not recommended ${ }^{43}$; transcatheter thrombolytic therapy was found in a separate randomized trial to be equivalent to fibrin sheath stripping. ${ }^{46}$ Despite the mixed efficacy of these techniques reported in the literature, most operators advocate beginning with transcatheter lytic therapy as the least invasive intervention, followed by catheter exchange with or without balloon disruption of the fibrin sheath.

For implanted ports that are resistant to tPA instillation, fibrin sheath stripping can be performed. From a common femoral vein access, a snare is advanced through the inferior vena cava and right atrium to encircle and grasp the catheter with the snare at the catheters midportion ( - Fig. 8). With moderate tension and moderate force, the snare is pulled until it slips completely off the catheter. It is normal for the catheter to elongate due to stretching; however, care should be taken to avoid pulling excessively, since disconnection of the catheter from the port or catheter fracture can occur. This method has been demonstrated to achieve greater than $90 \%$ success rates. ${ }^{47}$ For catheter tips that are adherent to the wall, additional methods may be required for detachment to allow snaring. Success has also been reported by advancing a 0.018 " guidewire through a Huber needle into the port and catheter. Subsequently, the guidewire can be snared from a femoral access. Once through-and-through access has been achieved, a snare can be advanced over the guidewire to facilitate snaring of the catheter. ${ }^{48}$

If all attempts have failed, for port and nonport catheters, de novo catheter insertion is necessary. While the original port pocket or tunneled access site can often be reused, a new venous access site should be used. By doing so, it can be ensured that the catheter is not advanced through the old fibrin sheath resulting in early reocclusion. In cases of venous occlusion or stenosis around the catheter tip, the catheter can be exchanged for a longer one if a guidewire can be advanced beyond the occlusion. Central venography, via catheter venography, CT venography, or magnetic resonance (MR) venography, can also be performed to identify cases where catheter obstruction is due to central venous thrombosis or occlusion.

\section{Central Venous Catheter Dislodgement/Malposition}

Malpositioning is a complication of CVC placement that can occur after initial insertion, usually related to respiratory motion and various body positions such as with maximum arm abduction. For tunneled and nontunneled catheters, exchanging the catheter under fluoroscopy and advancing/ repositioning the catheter with a guidewire are the most common solutions. In one retrospective study, this maneuver had a success rate of $65 \%$ at the bedside and $100 \%$ under fluoroscopy. ${ }^{49}$ Another less invasive technique described for smaller catheters, such as single lumen ports (5-6F) or PICCs, is the use of a 1- to 3-mL syringe to rapidly infuse saline with a forceful injection into the catheter. ${ }^{50,51}$ For implanted port catheters with tip malpositioning, excision of the port with exchange for a new port with a well-positioned catheter is the most definitive option, although attempts to reposition the catheter can be performed using snares from a groin or neck access.

Dislodgement of tunneled catheters tends to occur in the early time interval prior to tissue ingrowth into the cuff. In the case of partial retraction with exposure of the cuff, catheter exchange is mandated because the cuff is contaminated once it is external to the tract. For complete catheter dislodgement,

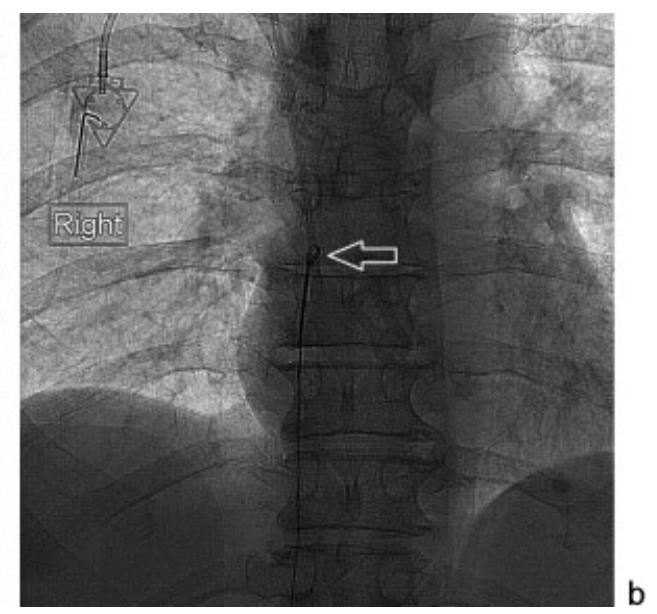
b

a

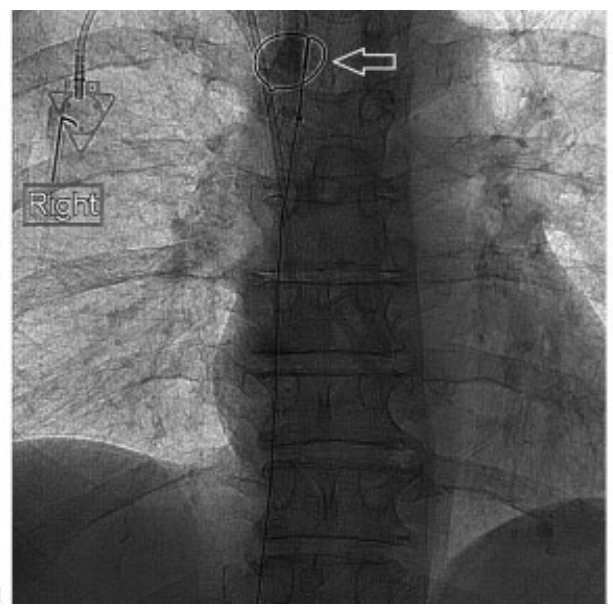

Fig. 8 Fibrin sheath stripping in patient from - Fig. 7. (a) A 15-mm gooseneck snare (arrow) is advanced over the catheter. (b) The snare is tightened (arrow) around the catheter and pulled downward to strip the fibrin sheath off of the catheter. 
reinsertion of the catheter through the prior tract can be attempted. This technique has been well described as safe and effective, particularly within the first 24 hours after dislodgment. In a retrospective study of 49 patients who underwent 57 recannulation procedures, an overall technical success rate of $86 \%$ was observed, with rates of $100 \%$ for catheters replaced within the first 12 hours and $64 \%$ for catheters that were replaced after 24 hours. ${ }^{52}$ A second, smaller study had similar findings in 24 patients with a success rate of $88 \%$ within the first 24 hours and $44 \%$ after 24 hours. $^{22}$ Of course, the alternative is to insert a de novo catheter.

\section{Conclusion}

Interventional radiologists place catheters for a variety of different indications. Unfortunately, catheters can be complicated by pericatheter leakage, obstruction, dislodgement, migration, malposition, and infection. In fact, catheter dysfunction is one of the most common reasons for a repeat intervention in interventional radiology. As patients live longer with catheters, it is important for the interventional radiologist not only to be aware of these problems, but also to have a thorough understanding of their etiology and potential solutions.

\section{References}

1 Barkmeier JM, Trerotola SO, Wiebke EA, et al. Percutaneous radiologic, surgical endoscopic, and percutaneous endoscopic gastrostomy/gastrojejunostomy: comparative study and cost analysis. Cardiovasc Intervent Radiol 1998;21(4):324-328

2 Silas AM, Pearce LF, Lestina LS, et al. Percutaneous radiologic gastrostomy versus percutaneous endoscopic gastrostomy: a comparison of indications, complications and outcomes in 370 patients. Eur J Radiol 2005;56(1):84-90

3 Wollman B, D'Agostino HB, Walus-Wigle JR, Easter DW, Beale A. Radiologic, endoscopic, and surgical gastrostomy: an institutional evaluation and meta-analysis of the literature. Radiology 1995; 197(3):699-704

4 Kim CY, Engstrom BI, Horvath JJ, Lungren MP, Suhocki PV, Smith TP. Comparison of primary jejunostomy tubes versus gastrojejunostomy tubes for percutaneous enteral nutrition. J Vasc Interv Radiol 2013;24(12):1845-1852

5 Dewald CL, Hiette PO, Sewall LE, Fredenberg PG, Palestrant AM. Percutaneous gastrostomy and gastrojejunostomy with gastropexy: experience in 701 procedures. Radiology 1999;211(3): 651-656

6 Alivizatos V, Gavala V, Alexopoulos P, Apostolopoulos A, Bajrucevic $S$. Feeding tube-related complications and problems in patients receiving long-term home enteral nutrition. Indian J Palliat Care 2012;18(1):31-33

7 Crosby J, Duerksen D. A retrospective survey of tube-related complications in patients receiving long-term home enteral nutrition. Dig Dis Sci 2005;50(9):1712-1717

8 Coleman DL, King RN, Andrade JD. The foreign body reaction: a chronic inflammatory response. J Biomed Mater Res 1974;8(5): 199-211

9 Ségal D, Michaud L, Guimber D, Ganga-Zandzou PS, Turck D, Gottrand F. Late-onset complications of percutaneous endoscopic gastrostomy in children. J Pediatr Gastroenterol Nutr 2001;33(4): 495-500
10 McClave SA, Chang WK. Complications of enteral access. Gastrointest Endosc 2003;58(5):739-751

11 Lin LF, Ko KC, Tsai YM, Huang JS. Buried bumper syndromecomplication of percutaneous endoscopic gastrostomy. Zhonghua Yi Xue Za Zhi (Taipei) 2001;64(5):315-319

12 McClave SA, Jafri NS. Spectrum of morbidity related to bolster placement at time of percutaneous endoscopic gastrostomy: buried bumper syndrome to leakage and peritonitis. Gastrointest Endosc Clin N Am 2007;17(4):731-746

$13 \mathrm{Kim} \mathrm{CH}$, Nelson DK. Venting percutaneous gastrostomy in the treatment of refractory idiopathic gastroparesis. Gastrointest Endosc 1998;47(1):67-70

14 Herman LL, Hoskins WJ, Shike M. Percutaneous endoscopic gastrostomy for decompression of the stomach and small bowel. Gastrointest Endosc 1992;38(3):314-318

15 Kim CY, Patel MB, Miller MJ Jr, Suhocki PV, Balius A, Smith TP. Gastrostomy-to-gastrojejunostomy tube conversion: impact of the method of original gastrostomy tube placement. J Vasc Interv Radiol 2010;21(7):1031-1037

16 Lord LM. Restoring and maintaining patency of enteral feeding tubes. Nutr Clin Pract 2003;18(5):422-426

17 Marcuard SP, Finley JL, MacDonald KG. Large-bore feeding tube occlusion by yeast colonies. JPEN J Parenter Enteral Nutr 1993; 17(2):187-190

18 Stumpf JL, Kurian RM, Vuong J, Dang K, Kraft MD. Efficacy of a Creon delayed-release pancreatic enzyme protocol for clearing occluded enteral feeding tubes. Ann Pharmacother 2014;48(4):483-487

19 Fischer LS, Bonello JC, Greenberg E. Gastrostomy tube migration and gastric outlet obstruction following percutaneous endoscopic gastrostomy. Gastrointest Endosc 1987;33(5):381-382

20 Huang SY, Levine MS, Raper SE. Gastrocolic fistula with migration of feeding tube into transverse colon as a complication of percutaneous endoscopic gastrostomy. AJR Am J Roentgenol 2005;184 (3, Suppl):S65-S66

21 Friedmann R, Feldman H, Sonnenblick M. Misplacement of percutaneously inserted gastrostomy tube into the colon: report of 6 cases and review of the literature. JPEN J Parenter Enteral Nutr 2007;31(6):469-476

22 Collares FB, Faintuch S, Kim SK, Rabkin DJ. Reinsertion of accidentally dislodged catheters through the original track: what is the likelihood of success? J Vasc Interv Radiol 2010;21(6):861-864

23 Halkier BK, Ho CS, Yee AC. Percutaneous feeding gastrostomy with the Seldinger technique: review of 252 patients. Radiology 1989; 171(2):359-362

24 Saini S, Mueller PR, Gaa J, et al. Percutaneous gastrostomy with gastropexy: experience in 125 patients. AJR Am J Roentgenol 1990;154(5):1003-1006

25 Ryan JM, Hahn PF, Boland GW, McDowell RK, Saini S, Mueller PR. Percutaneous gastrostomy with T-fastener gastropexy: results of 316 consecutive procedures. Radiology 1997;203(2):496-500

26 Sydnor RH, Schriber SM, Kim CY. T-fastener migration after percutaneous gastropexy for transgastric enteral tube insertion. Gut Liver 2014;8(5):495-499

27 Morrison JJ, McVinnie DW, Suiter PA, de Quadros NM. Percutaneous jejunostomy: repeat access at the healed site of prior surgical jejunostomy with US and fluoroscopic guidance. J Vasc Interv Radiol 2012;23(12):1646-1650

28 Mueller PR, van Sonnenberg E, Ferrucci JT Jr. Percutaneous biliary drainage: technical and catheter-related problems in 200 procedures. AJR Am J Roentgenol 1982;138(1):17-23

29 Hamlin JA, Friedman M, Stein MG, Bray JF. Percutaneous biliary drainage: complications of 118 consecutive catheterizations. Radiology 1986;158(1):199-202

30 Anderson JK, Monga M. Management of the retained percutaneous nephrostomy catheter. JSLS 2006;10(1):126-128

31 Paul EM, Marcovich R, Lee BR, Smith AD. Choosing the ideal nephrostomy tube. BJU Int 2003;92(7):672-677 
32 Cazenave FL, Glass-Royal MC, Barth KH. Exchange of an obstructed loop nephrostomy catheter: technical note. Cardiovasc Intervent Radiol 1990;13(5):327-328

33 Lee AS, vanSonnenberg E, Wittich GR, Casola G. Exchange of occluded catheters with transcatheter and pericatheter maneuvers. Radiology 1987;163(1):273-274

34 Pollack HM, Banner MP. Replacing blocked or dislodged percutaneous nephrostomy and ureteral stent catheters. Radiology 1982; 145(1):203-205

$35 \mathrm{Ng}$ CK, Yip SK, Sim LS, et al. Outcome of percutaneous nephrostomy for the management of pyonephrosis. Asian J Surg 2002; 25(3):215-219

36 Schuster MR, Crummy AB, Wojtowycz MM, McDermott JC. Abdominal abscesses associated with enteric fistulas: percutaneous management. J Vasc Interv Radiol 1992;3(2):359-363

37 Baskin JL, Pui CH, Reiss U, et al. Management of occlusion and thrombosis associated with long-term indwelling central venous catheters. Lancet 2009;374(9684):159-169

38 Rasmussen RL. The catheter-challenged patient and the need to recognize the recurrently dysfunctional tunneled dialysis catheter. Semin Dial 2010;23(6):648-652

39 Brismar B, Hårdstedt C, Jacobson S. Diagnosis of thrombosis by catheter phlebography after prolonged central venous catheterization. Ann Surg 1981;194(6):779-783

40 Nadolski G, Shlansky-Goldberg RD, Stavropoulos SW, Soulen MC, Farrelly C, Trerotola SO. Chest radiograph-based algorithm for managing malfunctioning ports. J Vasc Interv Radiol 2013;24(9): 1337-1342

41 Haymond J, Shalansky K, Jastrzebski J. Efficacy of low-dose alteplase for treatment of hemodialysis catheter occlusions. J Vasc Access 2005;6(2):76-82

42 Savader SJ, Ehrman KO, Porter DJ, Haikal LC, Oteham AC. Treatment of hemodialysis catheter-associated fibrin sheaths by rt-PA infusion: critical analysis of 124 procedures. J Vasc Interv Radiol 2001; 12(6):711-715
43 Merport M, Murphy TP, Egglin TK, Dubel GJ. Fibrin sheath stripping versus catheter exchange for the treatment of failed tunneled hemodialysis catheters: randomized clinical trial. J Vasc Interv Radiol 2000;11(9):1115-1120

44 Beathard GA. Dysfunction of new catheters by old fibrin sheaths. Semin Dial 2004;17(3):243-244

45 Engstrom BI, Horvath JJ, Stewart JK, et al. Tunneled internal jugular hemodialysis catheters: impact of laterality and tip position on catheter dysfunction and infection rates. J Vasc Interv Radiol 2013; 24(9):1295-1302

46 Gray RJ, Levitin A, Buck D, et al. Percutaneous fibrin sheath stripping versus transcatheter urokinase infusion for malfunctioning wellpositioned tunneled central venous dialysis catheters: a prospective, randomized trial. J Vasc Interv Radiol 2000;11(9):1121-1129

47 Heye S, Maleux G, Goossens GA, Vaninbroukx J, Jerôme M, Stas M. Feasibility and safety of endovascular stripping of totally implantable venous access devices. Cardiovasc Intervent Radiol 2012; 35(3):607-612

48 Sobolevsky S, Sheiman RG, Faintuch S, Perry L. Femoral-to-port through-and-through wire access to reestablish subcutaneous port function. AJR Am J Roentgenol 2007;188(4):1047-1049

49 Trerotola SO, Thompson S, Chittams J, Vierregger KS. Analysis of tip malposition and correction in peripherally inserted central catheters placed at bedside by a dedicated nursing team. J Vasc Interv Radiol 2007;18(4):513-518

50 Jin J, Chen C, Zhao R, Li A, Shentu Y, Jiang N. Repositioning techniques of malpositioned peripherally inserted central catheters. J Clin Nurs 2013;22(13-14):1791-1804

51 Sharpe EL. Repositioning techniques for malpositioned neonatal peripherally inserted central catheters. Adv Neonatal Care 2010; 10(3):129-132

52 Saad NE, Saad WE, Davies MG, Waldman DL. Replacement of inadvertently discontinued tunneled jugular high-flow central catheters with tract recannulation: technical results and outcome. J Vasc Interv Radiol 2008;19(6):890-896 